\title{
Sonication-Triggered Rolling of Janus Porous Nanomembranes for Electrochemical Sensing of Dopamine and Ascorbic Acid
}

\author{
Fei Mal, Bin Yang ${ }^{2}$, Zhe Zhao ${ }^{1}$, Yuting Zhao ${ }^{1}$, Ruobing Pan ${ }^{1}$, Dingrun Wang ${ }^{1}$, Ye \\ Kong ${ }^{1}$, Yimeng Chen ${ }^{l}$, Gaoshan Huang ${ }^{1, *}$, Jilie Kong ${ }^{2}$, Yongfeng Mei ${ }^{1, *}$ \\ ${ }^{1}$ Department of Materials Science, Fudan University, 220 Handan Road, Shanghai \\ 200433, People's Republic of China \\ ${ }^{2}$ Department of Chemistry, Fudan University, Shanghai 200433, People's Republic \\ of China
}

\section{Corresponding Authors}

*Gaoshan Huang, Department of Materials Science, Fudan University, Shanghai 200433, P. R. China; Email: gshuang@fudan.edu.cn

*Yongfeng Mei, Department of Materials Science, Fudan University, Shanghai 200433, P. R. China; Email: yfm@fudan.edu.cn 

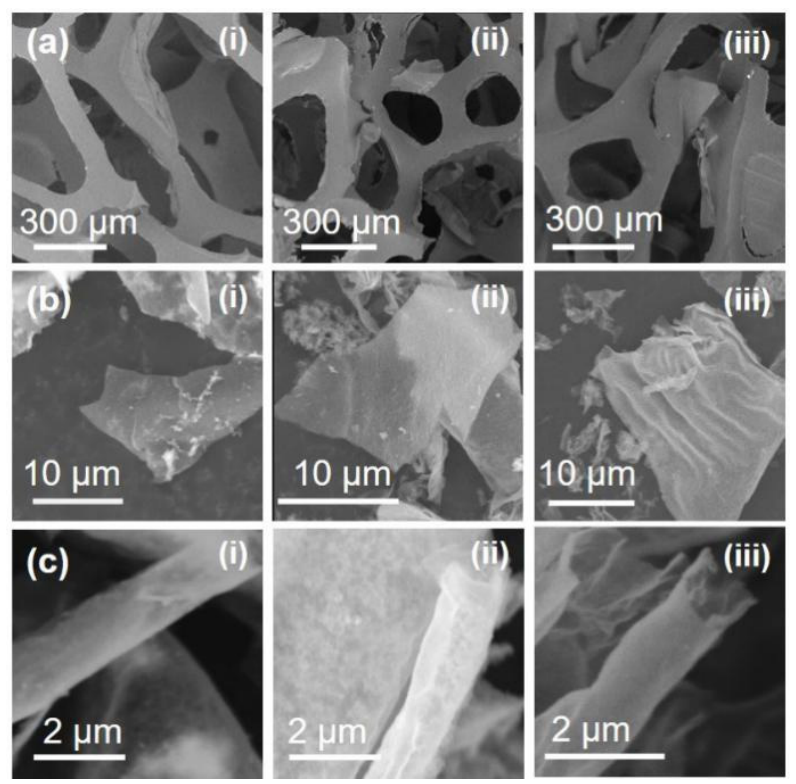

Figure S1. SEM images of (a) sponge template coated with $\mathrm{TiO}_{2}$ nanomembrane, (b) free-standing $\mathrm{TiO}_{2}$ nanomembranes, and (c) $\mathrm{TiO}_{2}$ microtubes. Panels (i), (ii), and (iii) correspond to $\mathrm{TiO}_{2}$ nanomembrane made from 100, 200, and 300 ALD cycles respectively. 

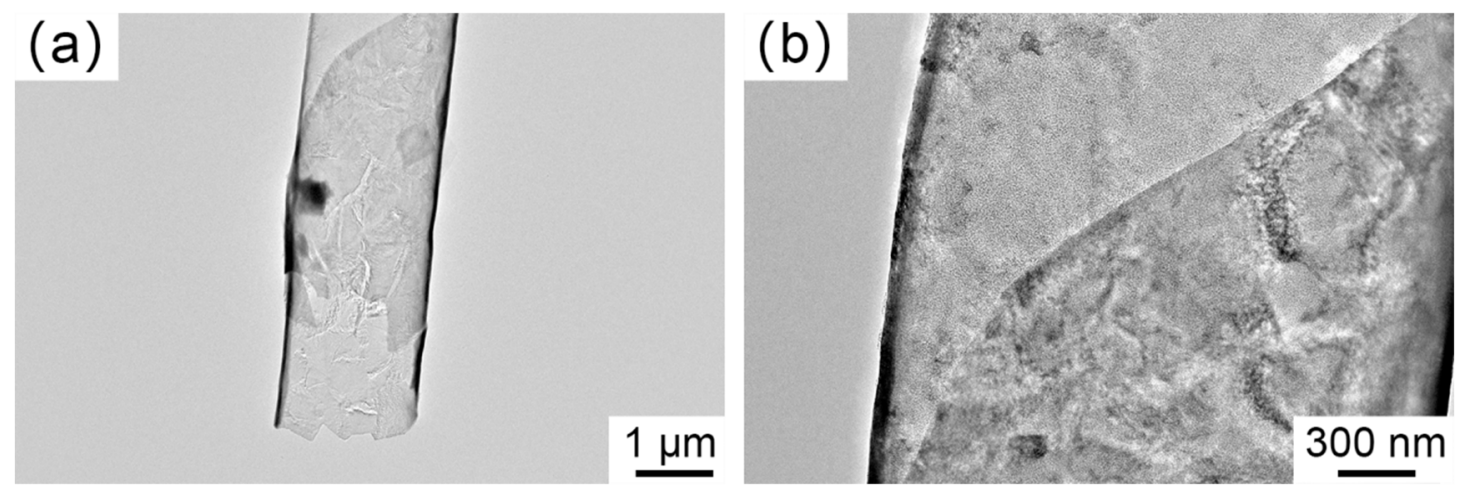

Figure S2. TEM images of microtube with various magnification. 

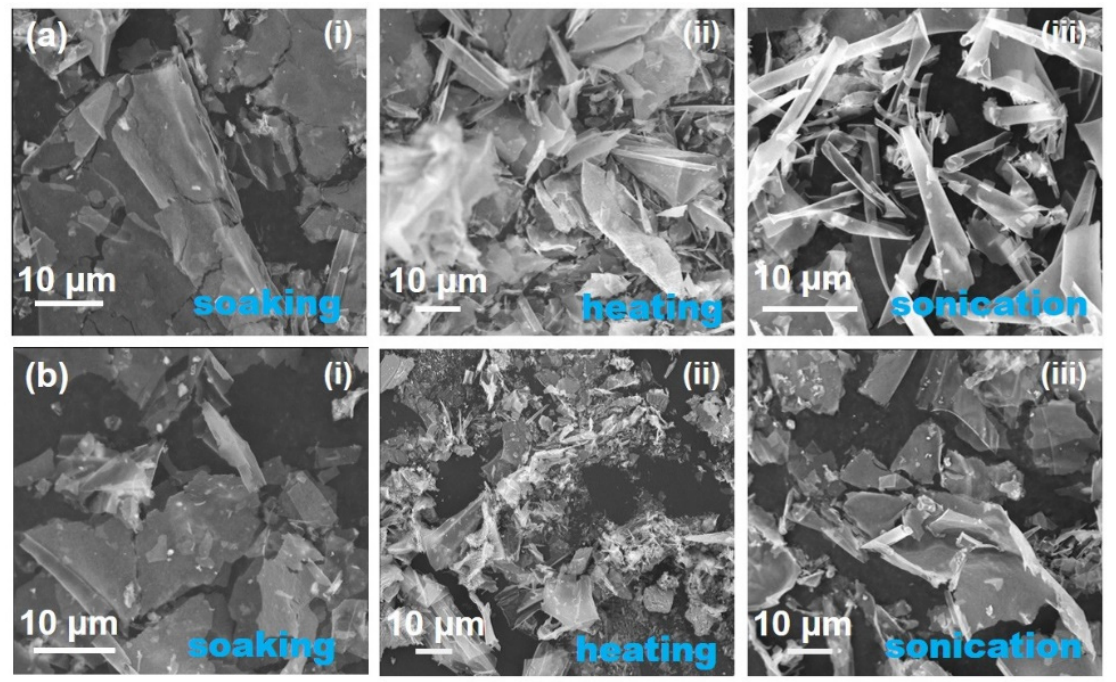

Figure S3. (a) SEM images of $\mathrm{TiO}_{2}$ nanomembranes (400 ALD cycles) in water after soaking (i, $24 \mathrm{~h}, 20^{\circ} \mathrm{C}$ ), heating (ii, $4 \mathrm{~h}, 60^{\circ} \mathrm{C}$ ), and sonication (iii, $2 \mathrm{~h}, 100 \mathrm{kHZ}$ ). (b) SEM images of $\mathrm{TiO}_{2}$ nanomembranes (400 ALD cycles) in hexane after soaking (i, 24 h, $20^{\circ} \mathrm{C}$ ), heating (ii, $4 \mathrm{~h}, 60^{\circ} \mathrm{C}$ ), and sonication (iii, $2 \mathrm{~h}, 100 \mathrm{kHZ}$ ). 

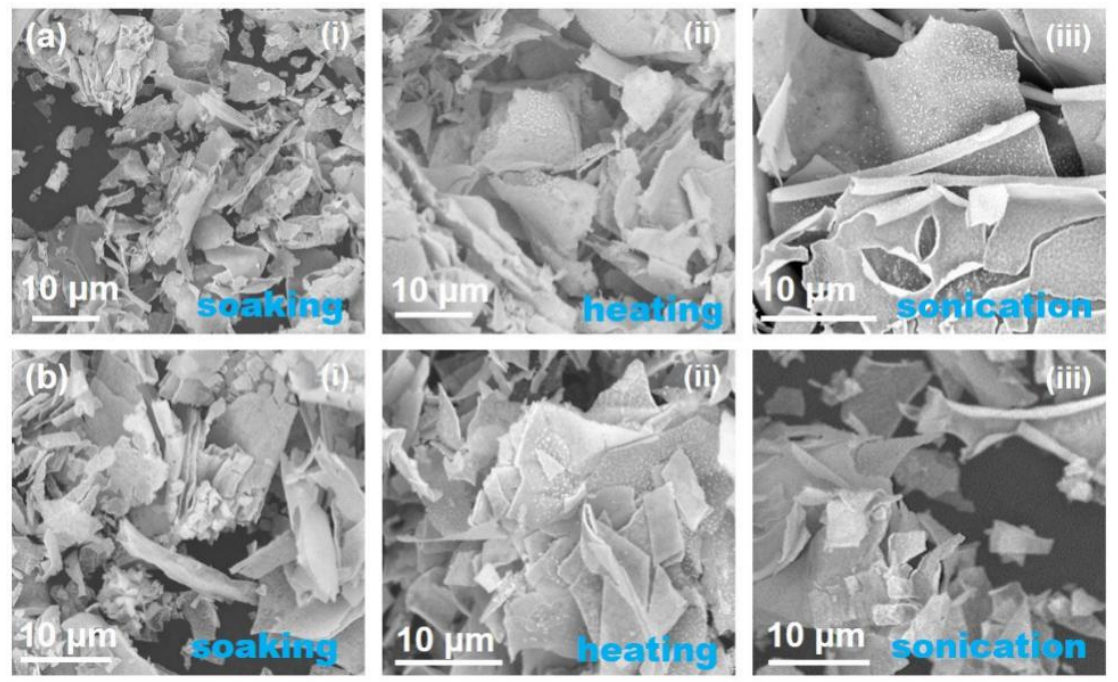

Figure S4. (a) SEM images of $\mathrm{ZnO}$ nanomembranes (200 ALD cycles) in water after soaking (i, $24 \mathrm{~h}, 20^{\circ} \mathrm{C}$ ), heating (ii, $4 \mathrm{~h}, 60^{\circ} \mathrm{C}$ ), and sonication (iii, $2 \mathrm{~h}, 100 \mathrm{kHZ}$ ). (b) SEM images of $\mathrm{ZnO}$ nanomembranes (200 ALD cycles) in hexane after soaking (i, 24 h, $20^{\circ} \mathrm{C}$ ), heating (ii, $4 \mathrm{~h}, 60^{\circ} \mathrm{C}$ ), and sonication (iii, $2 \mathrm{~h}, 100 \mathrm{kHZ}$ ). 

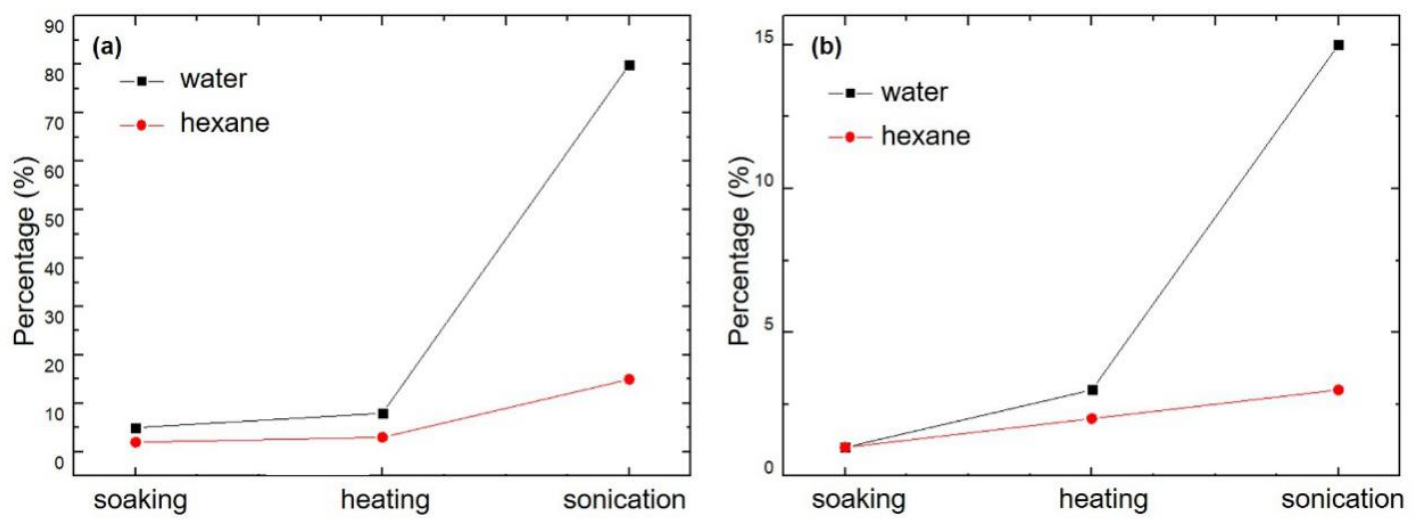

Figure S5. (a) Statistics of microtube formation. $\mathrm{TiO}_{2}$ nanomembranes (400 ALD cycles) were treated by soaking, heating, and sonication. Water and hexane were used as solvents. (b) Statistics of microtube formation. $\mathrm{ZnO}$ nanomembranes (200 ALD cycles) were treated by soaking, heating, and sonication. Water and hexane were used as solvents. 


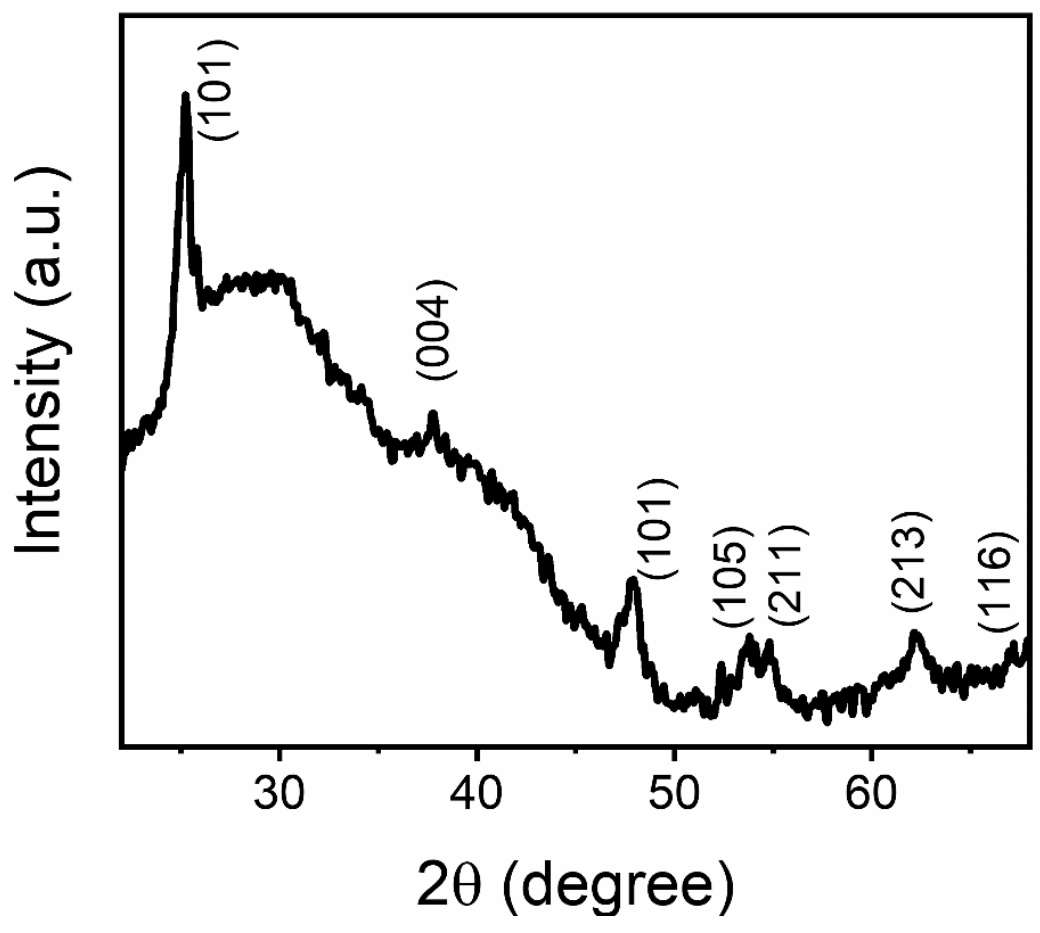

Figure S6. XRD pattern of $\mathrm{TiO}_{2}$ microtubes sample. 

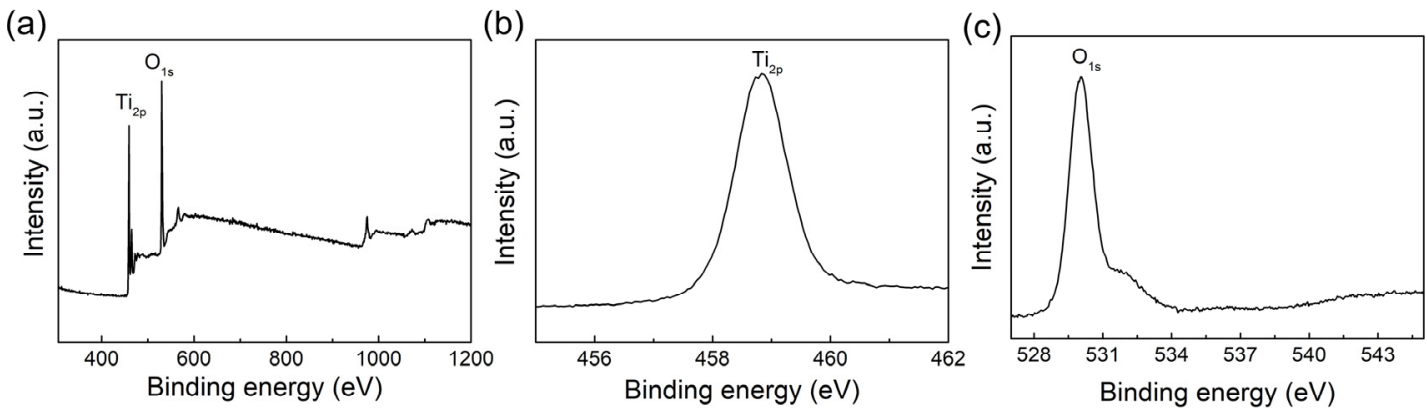

Figure S7. (a) XPS survey scan of $\mathrm{TiO}_{2}$ microtubes. (b) High-resolution $\mathrm{Ti} 2 \mathrm{p}$ spectrum and (c) O1s spectrum. 

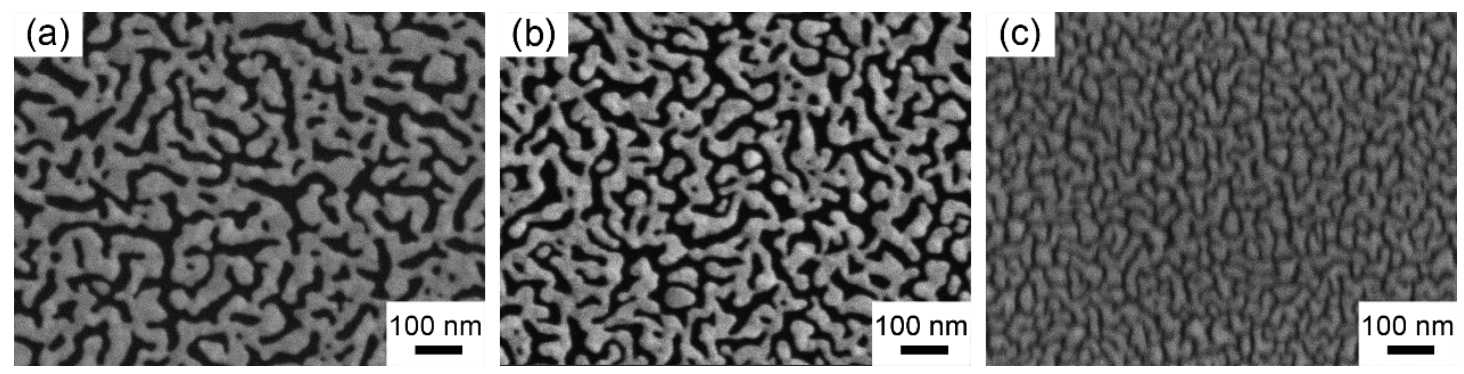

Figure S8. SEM images of $\mathrm{Au}$ nanoparticles deposited on $\mathrm{TiO}_{2}$ nanomembrane with a thickness of (a) $2 \mathrm{~nm}$, (b) $4 \mathrm{~nm}$ and (c) $6 \mathrm{~nm}$. 


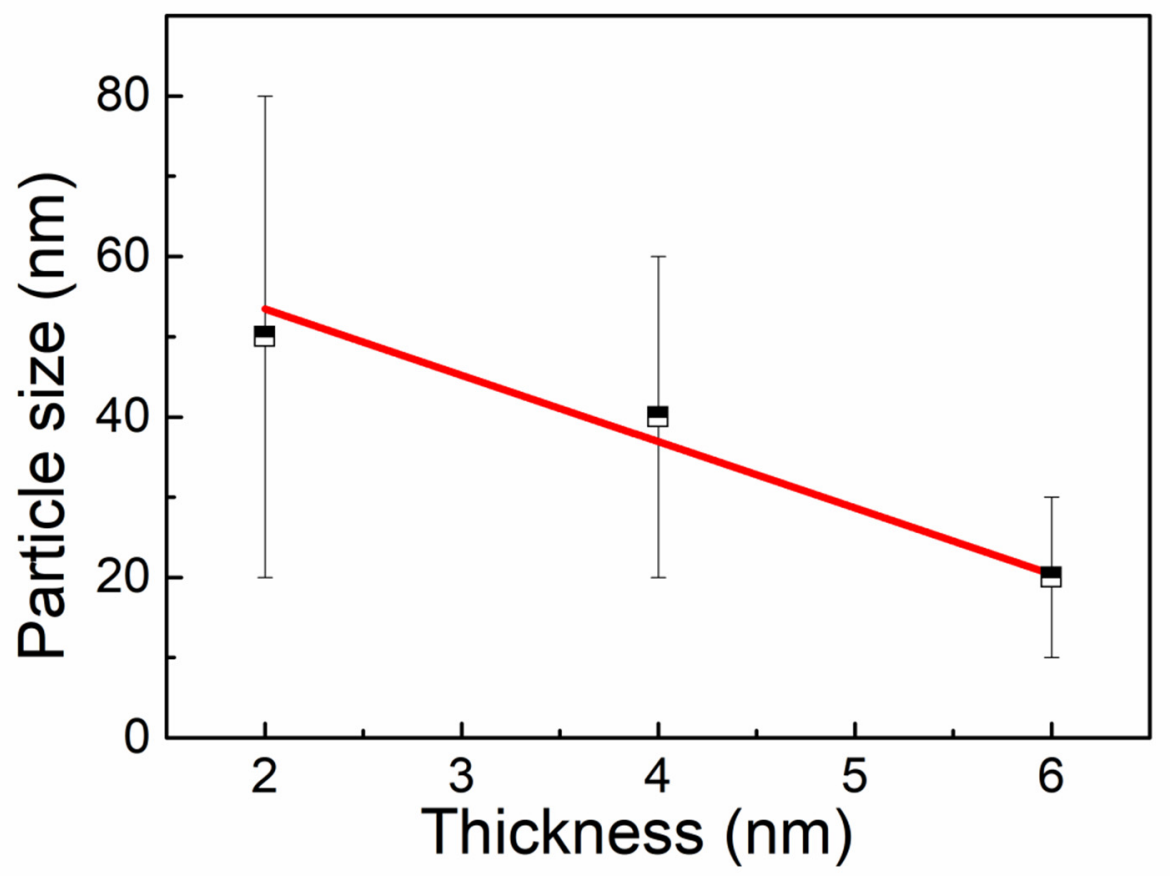

Figure S9. The particle size distributions of Au with various thicknesses. 

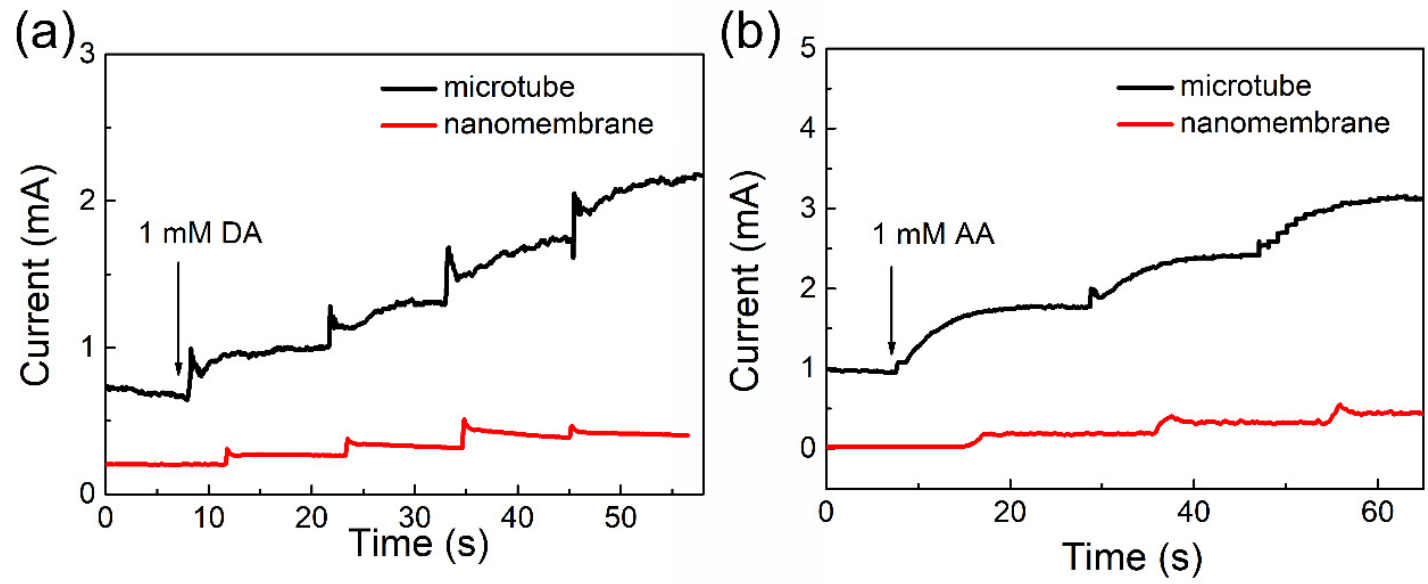

Figure S10. The i-t curves of the nanomembrane-based and the microtube-based sensors with successive addition of (a) $1 \mathrm{mM}$ DA and (b) $1 \mathrm{mM} \mathrm{AA}$. 

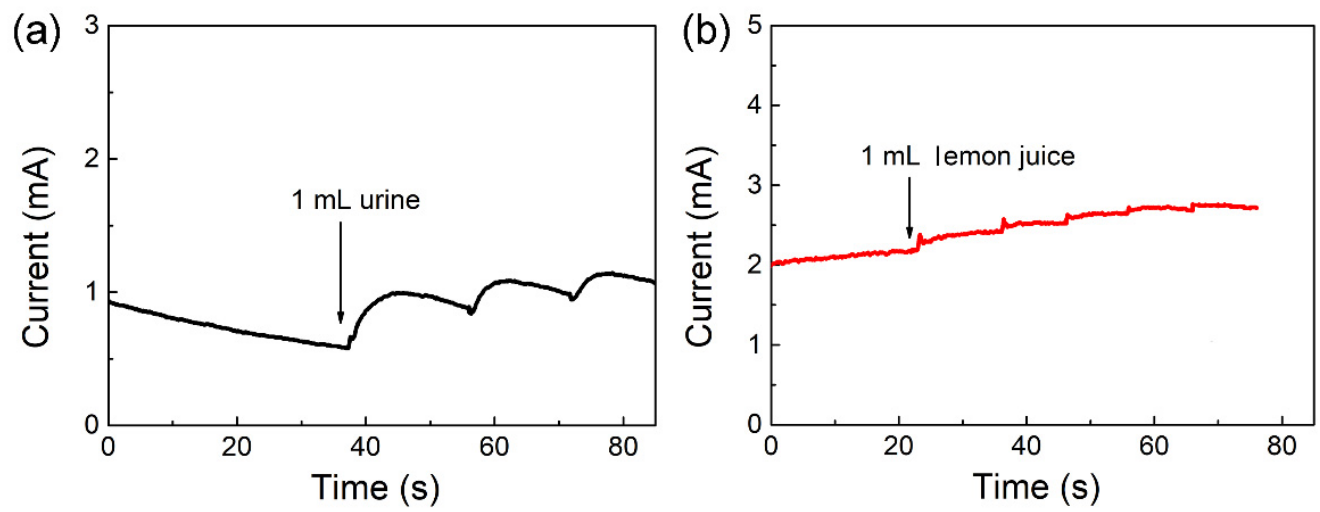

Figure S11. The i-t curves of microtube with successive addition of (a) $1 \mathrm{~mL}$ urine and (b) $1 \mathrm{~mL}$ lemon juice. 
(a)

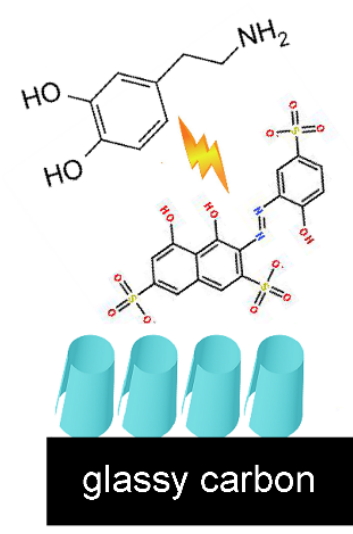

(b)

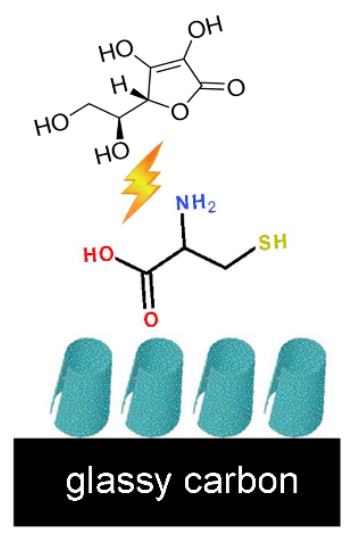

(c)

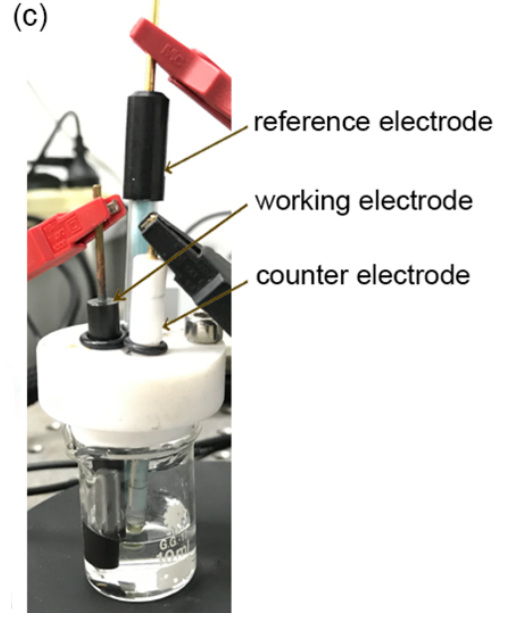

Figure S12. (a) and (b) Schematics of working electrodes. (c) Photograph of a sensor device. 
Table S1. Fitting parameters of $\mathrm{TiO}_{2}$ microtube with $\mathrm{Au}$ particles derived from EIS curves.

\begin{tabular}{ccc}
\hline $\mathrm{R}(\Omega)$ & $\mathrm{C}(\mathrm{F})$ & $\mathrm{W}\left(\Omega^{-1} \mathrm{~s}^{0.5}\right)$ \\
\hline 575.8 & 0.00005 & 0.00005 \\
\hline
\end{tabular}

